\title{
Spontaneous cycloidal order mediating a spin- reorientation transition in a polar metal
}

\author{
C. D. Dashwood, L. S. I. Veiga, Q. Faure, J. G. Vale, D. G. Porter, S. \\ P. Collins, P. Manuel, D. D. Khalyavin, F. Orlandi, R. S. Perry, R. D. \\ Johnson, and D. F. McMorrow
}

\section{Published version information}

Citation: CD Dashwood et al. "Spontaneous cycloidal order mediating a spinreorientation transition in a polar metal." Phys Rev B 102, no. 18 (2020): 180410.

DOI: $\underline{10.1103 / P h y s R e v B .102 .180410}$

This version is made available in accordance with publisher policies. Please cite only the published version using the reference above. This is the citation assigned by the publisher at the time of issuing the APV. Please check the publisher's website for any updates. 


\title{
Spontaneous cycloidal order mediating a spin-reorientation transition in a polar metal
}

\author{
C. D. Dashwood $\odot,{ }^{1, *}$ L. S. I. Veiga $\odot,{ }^{1}$ Q. Faure,,${ }^{1}$ J. G. Vale, ${ }^{1}$ D. G. Porter,${ }^{2}$ S. P. Collins $\odot,{ }^{2}$ P. Manuel,,${ }^{3}$ D. D. Khalyavin, ${ }^{3}$ \\ F. Orlandi ${ }^{3}{ }^{3}$ R. S. Perry, ${ }^{1}$ R. D. Johnson, ${ }^{4}$ and D. F. McMorrow ${ }^{1, \dagger}$ \\ ${ }^{1}$ London Centre for Nanotechnology and Department of Physics and Astronomy, University College London, \\ London WC1E 6BT, United Kingdom \\ ${ }^{2}$ Diamond Light Source, Harwell Science and Innovation Campus, Didcot, Oxfordshire OX11 ODE, United Kingdom \\ ${ }^{3}$ ISIS Neutron and Muon Source, STFC Rutherford Appleton Laboratory, Didcot, Oxfordshire OX11 OQX, United Kingdom \\ ${ }^{4}$ Department of Physics and Astronomy, University College London, London WC1E 6BT, United Kingdom
}

(Received 18 June 2020; revised 5 November 2020; accepted 12 November 2020; published 25 November 2020)

\begin{abstract}
We show how complex modulated order can spontaneously emerge when magnetic interactions compete in a metal with polar lattice distortions. Combining neutron and resonant $\mathrm{x}$-ray scattering with symmetry analysis, we reveal that the spin reorientation in $\mathrm{Ca}_{3} \mathrm{Ru}_{2} \mathrm{O}_{7}$ is mediated by a magnetic cycloid whose eccentricity evolves smoothly but rapidly with temperature. We find the cycloid to be highly sensitive to magnetic fields, which appear to continuously generate higher harmonic modulations. Our results provide a unified picture of the rich magnetic phases of this correlated, multiband polar metal.
\end{abstract}

DOI: 10.1103/PhysRevB.102.180410

The combination of polar distortions and magnetic order leads to the celebrated functionality of magnetoelectric multiferroics [1,2]. Of particular interest are the magnetically driven ferroelectrics, in which a macroscopic electric polarization is induced by noncentrosymmetric magnetic ordering [3]. In frustrated magnets such as $\mathrm{TbMnO}_{3}$ [4], for instance, inversion symmetry is broken by complex spiral ordering which itself arises from competing magnetic interactions.

While conventional multiferroics are insulating, attention has recently been focused on polar metals, in which polar distortions and conduction electrons coexist $[5,6]$. The bilayer ruthenate $\mathrm{Ca}_{3} \mathrm{Ru}_{2} \mathrm{O}_{7}$ is a particularly rare example of a polar metal that also magnetically orders [7-9]. Tilts and rotations of the $\mathrm{RuO}_{6}$ unlock polar displacements of the $\mathrm{Cu}$ and $\mathrm{O}$ ions, which are not screened as only Ru contributes to the Fermi surface [10]. Alongside polar domains which can be switched ferroelastically [10], $\mathrm{Ca}_{3} \mathrm{Ru}_{2} \mathrm{O}_{7}$ demonstrates magnetic switching: a thermally driven spin-reorientation transition (SRT). At $T_{S}=48 \mathrm{~K}$ the collinear Ru moments undergo a reorientation from the $\boldsymbol{b}\left[\mathrm{AFM}_{\mathrm{b}}\right.$, see Fig. 1(b)] to $\boldsymbol{a}$ axis $\left[\mathrm{AFM}_{\mathrm{a}}\right.$, Fig. 1(d)] [11,12]. In both phases the spins are aligned ferromagnetically within each bilayer and antiferromagnetically between the bilayers, with propagation vector $(0,0,1)[8,11,12]$. Due to the interplay of electronic correlations and spin-orbit coupling, the SRT is intricately coupled to the crystal structure and fermiology, coinciding with an isostructural change in the lattice parameters [8] and an increase in the resistivity caused by a gapping of most of the Fermi surface [13-15].

In this Rapid Communication, we report the inverse of the magnetically driven ferroelectric mechanism in $\mathrm{Ca}_{3} \mathrm{Ru}_{2} \mathrm{O}_{7}$ :

\footnotetext{
*cameron.dashwood.17@ucl.ac.uk

†.mcmorrow@ucl.ac.uk
}

the formation of modulated order driven by competing magnetic interactions in the presence of structurally broken inversion symmetry. Exploiting the complementarity of neutron and resonant $x$-ray scattering, we reveal a magnetic cycloid which evolves continuously between the collinear end states over a remarkably small temperature range to mediate the SRT. Symmetry analysis shows that the cycloid is stabilized by a uniform Dzyaloshinskii-Moriya interaction (DMI), stemming from spin-orbit coupling and activated by the small polar distortions, which competes with easy-axis anisotropies. We show how the frustration that renders the cycloid sensitive to temperature also allows it to be delicately tuned by magnetic field, under which higher harmonic modulations of the fundamental order appear to be generated. This necessitates a reinterpretation of an incommensurate structure previously observed under field [16], unifying it with the magnetic response to doping [17-19]. Our results thus demonstrate a transition at the juncture of polar metals, spin-orbit-coupled systems, and magnetic frustration.

High-quality single crystals of $\mathrm{Ca}_{3} \mathrm{Ru}_{2} \mathrm{O}_{7}$ were grown by the floating zone method, and characterized by x-ray powder diffraction, resistivity measurements, and energy dispersive $\mathrm{x}$-ray spectrometry. Twin domains were identified with polarized light microscopy, and single-domain pieces were cut from larger crystals using a wire saw. Measurements were performed on multiple crystals from different growth batches with consistent results. The neutron and x-ray data presented below are all from the same crystal, which was aligned by Laue diffraction. Neutron scattering measurements were performed at the WISH instrument of the ISIS Neutron and Muon Source [20], and resonant x-ray scattering measurements at beamline I16 of the Diamond Light Source. Further experimental details can be found in the Supplemental Material [21].

We first report the discovery of bulk incommensurate order in pristine $\mathrm{Ca}_{3} \mathrm{Ru}_{2} \mathrm{O}_{7}$ from neutron scattering measurements. 
(a)

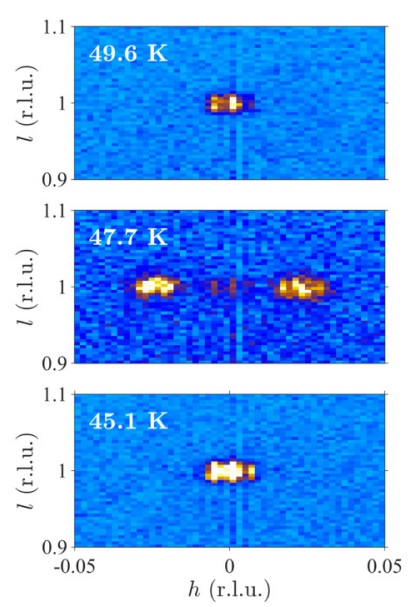

(b)

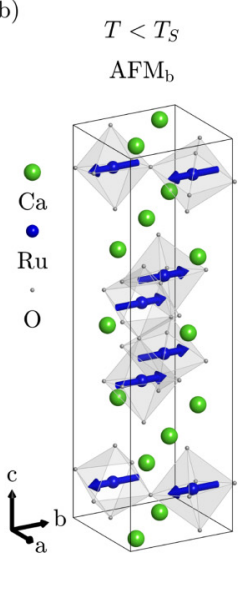

(c)

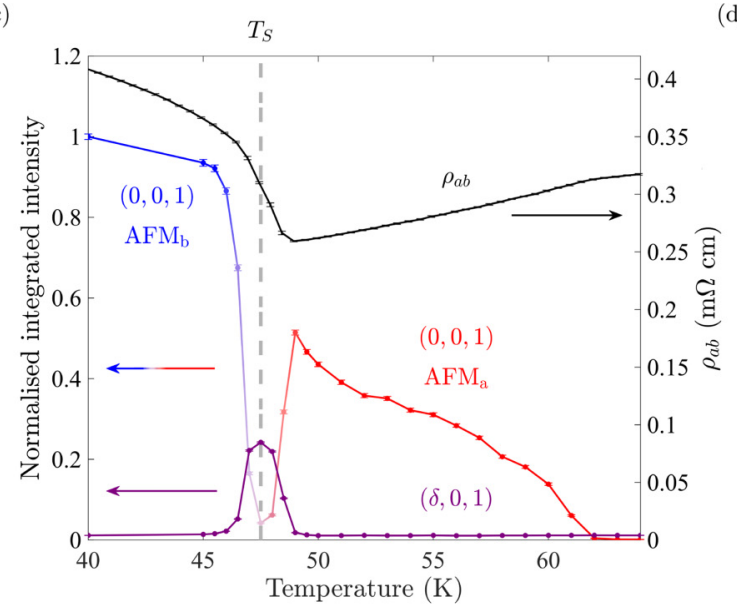

(d)

$$
\begin{aligned}
& T>T_{S} \\
& \mathrm{AFM}_{\mathrm{a}}
\end{aligned}
$$

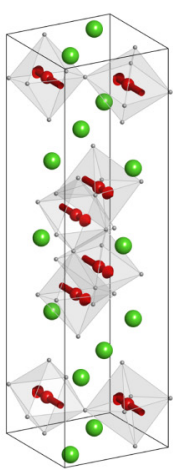

FIG. 1. Incommensurate satellites at the spin reorientation from neutron scattering. (a) Reciprocal space maps showing satellite peaks $( \pm \delta, 0,1)$ around the $(0,0,1)$ magnetic peak over a narrow temperature range around $T_{S}=48 \mathrm{~K}$. (b) Crystal and magnetic structure of $\mathrm{Ca}_{3} \mathrm{Ru}_{2} \mathrm{O}_{7}$ below $T_{S}$ with collinear spins pointing along the $\boldsymbol{b}$ axis. (c) Integrated intensity of the $(0,0,1)$ (blue to red, with blue representing the AFM and red the $\mathrm{AFM}_{\mathrm{a}}$ phase) and $(+\delta, 0,1)$ (purple) peaks as a function of temperature, plotted alongside the in-plane resistivity $\rho_{a b}$ (black). (d) Crystal and magnetic structure of $\mathrm{Ca}_{3} \mathrm{Ru}_{2} \mathrm{O}_{7}$ for $T_{S}<T<T_{N}$ with spins pointing along the $\boldsymbol{a}$ axis.

Figure 1(a) shows reciprocal-space maps at temperatures around $T_{S}$. Well above and below $T_{S}$ (top and bottom panels) a single peak can be seen at $\boldsymbol{q}=(0,0,1)$ reciprocal lattice units (r.l.u.) which arises from the known $\mathrm{AFM}_{\mathrm{a}}$ and $\mathrm{AFM}_{\mathrm{b}}$ structures. As unpolarized neutron scattering is sensitive to the component of the moment perpendicular to $\boldsymbol{q}$, we are sensitive to the full moment in both phases and the lower intensity in the former is indicative of a smaller moment, consistent with previous reports [12]. Strikingly, close to $T_{S}$ (middle panel) the central peak is strongly suppressed, and satellites can be seen at incommensurate positions $( \pm \delta, 0,1)$ with $\delta \approx 0.023$ r.l.u. Satellite peaks closely spaced around a commensurate position are indicative of a long-range modulation of the nuclear and/or magnetic structure, such as charge/spin density wave phases [22,23] or spiral magnetic structures [24,25]. In this case, the transfer of intensity from the commensurate to satellite peaks suggests a common magnetic origin, while their comparable widths indicate long correlation lengths. Comparing the integrated intensity of the $(0,0,1)$ and $(\delta, 0,1)$ peaks to the in-plane resistivity $\rho_{a b}$ in Fig. 1(c), it can be seen that the boundaries of the incommensurate phase correspond to changes of slope in $\rho_{a b}$, revealing how the magnetic structure is intimately linked to the electronic behavior.

Having established the existence of an incommensurate phase in the vicinity of the SRT, we now turn to resonant X-ray scattering to unravel its nature. Tuning the incident $x$ rays to the $\mathrm{Ru} L_{2}$ absorption edge causes a resonant enhancement of the scattered intensity, which combined with a polarization analysis of the scattered beam results in an element-selective probe of long-range magnetic order. Figure 2(a) shows rocking scans of the $(0,0,5)$ peak in two orthogonal polarization channels. X rays polarized in the scattering plane are denoted $\pi$, and those normal to it $\sigma$ [see Fig. 2(b)]. For incident $\sigma \mathrm{x}$ rays, resonant scattering from a magnetic moment occurs only in the crossed $\sigma-\pi^{\prime}$ polarization channel [26], so the dominant intensity in this channel verifies the magnetic origin of the peak. The remnant intensity in the $\sigma-\sigma^{\prime}$ channel is due to leakage through the analyzer (see the Supplemental Material [21]).
In contrast to neutron scattering, resonant $\mathrm{x}$-ray scattering in $\sigma-\pi^{\prime}$ is sensitive to the component of the moment parallel to $\boldsymbol{k}_{\boldsymbol{f}}$. More information about the magnetic structure can therefore be obtained by rotating the sample through an azimuthal angle $\Psi$ in order to vary the component projected along $\boldsymbol{k}_{\boldsymbol{f}}$ [see Fig. 2(b)]. Azimuthal dependences of the $(0,0,5)$ peak are shown in Fig. 2(c). For basal-plane collinear structures, calculation of the resonant cross section gives an intensity $\propto \cos ^{2}(\Psi+\phi)$ [solid lines in Fig. 2(c)] where $\phi$ is the rotation of the moments away from the $\boldsymbol{a}$ axis [11]. At $40.4 \mathrm{~K}$ it can be seen that $\phi=90^{\circ}$ and the moments are along $\boldsymbol{b}$, while at $55.6 \mathrm{~K} \phi=0^{\circ}$ and the moments are along $\boldsymbol{a}$. The upper and lower panels in Fig. 2(d) show $h$ scans through $(0,0,5)$ as a function of temperature for two azimuths, sensitive to the $\boldsymbol{b}\left(\Psi=-90^{\circ}\right)$ and $\boldsymbol{a}\left(\Psi=-180^{\circ}\right)$ components of the moments, respectively. The spin reorientation is clearly identified by the transfer of commensurate intensity between the azimuths. The satellite peaks are also visible at both azimuths, and intriguingly have subtle temperature dependences to their wave vectors and intensities. We now analyze these satellites in more detail.

Figure 3(a) shows $h$ scans in both polarization channels, confirming the magnetic origin of the satellites. In order to determine the structure of the incommensurate phase we performed a detailed investigation of the azimuthal dependence of the satellites. Representative dependences are shown in Fig. 3(b). All of the dependences are sinusoidal, but show dramatic changes in peak-to-peak amplitude and phase, evidencing a remarkable evolution of the structure over a small temperature window. Unlike in the commensurate phases where the intensity goes to zero when $\boldsymbol{k}_{\boldsymbol{f}}$ is perpendicular to $\boldsymbol{m}$, here we see a finite intensity at all $\Psi$. This is a clear indication of a noncollinear rotating structure, with a component of the moment always parallel to $\boldsymbol{k}_{f}$. We calculated the cross section for all possible modulated states using the MAGNETIX package [27] and found that a cycloid with moments rotating in the $\boldsymbol{a}-\boldsymbol{b}$ plane, maintaining the ferromagnetic coupling within bilayers and antiferromagnetic 


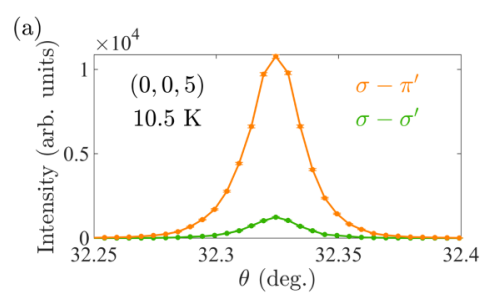

(b)

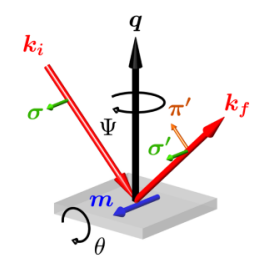

(c)

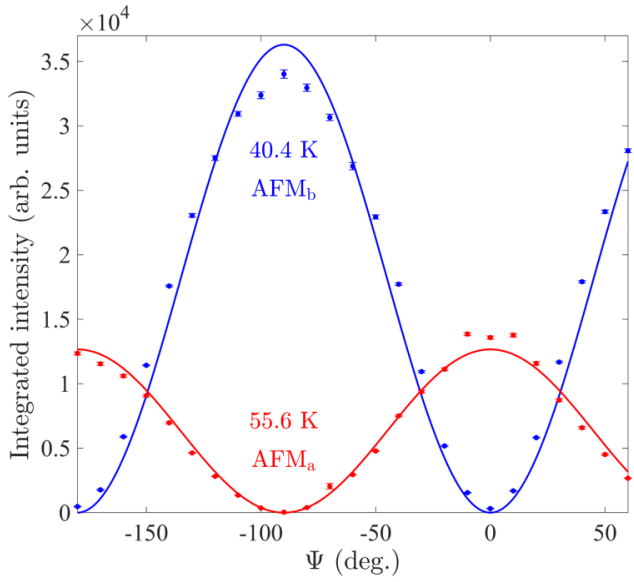

(d)
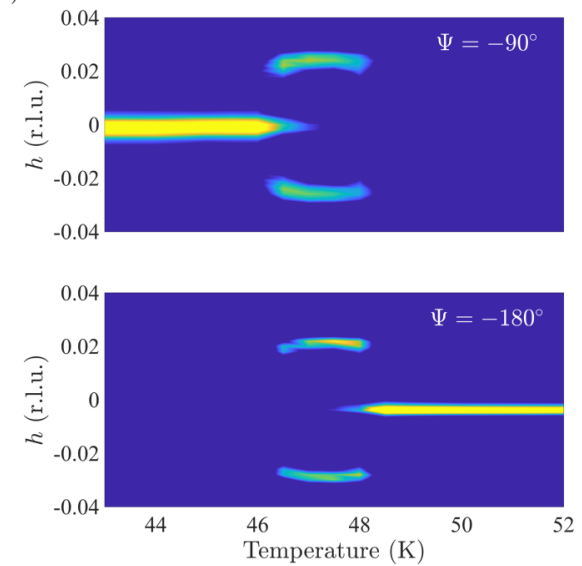

FIG. 2. Spin reorientation from resonant x-ray scattering. (a) Rocking curves of the $(0,0,5)$ peak at $10.5 \mathrm{~K}$ in the $\sigma-\pi^{\prime}$ (orange) and $\sigma-\sigma^{\prime}$ (green) polarization channels, confirming its magnetic origin. (b) Experimental geometry, showing incident x rays with wave vector $\boldsymbol{k}_{\boldsymbol{i}}$ (red arrow) scattering from a magnetic moment $\boldsymbol{m}$ (blue arrow) to wave vector $\boldsymbol{k}_{\boldsymbol{f}}$. The incident $\mathrm{x}$ rays are polarized horizontally, normal to the scattering plane ( $\sigma$ polarized, green arrow), and the scattered x rays are polarized either in the scattering plane $\left(\pi^{\prime}\right.$, orange) or normal it $\left(\sigma^{\prime}\right.$, green). The azimuth $\Psi$ is varied by rotating the sample around the scattering vector $\boldsymbol{q}=\boldsymbol{k}_{\boldsymbol{f}}-\boldsymbol{k}_{\boldsymbol{i}}$ (black arrow). (c) Azimuthal scans of the $(0,0,5)$ peak above $\left(55.6 \mathrm{~K}\right.$, red) and below $\left(40.4 \mathrm{~K}\right.$, blue) $T_{S}$. (d) $h$ scans through $(0,0,5)$ as a function of temperature at $\Psi=-90^{\circ}($ top panel, sensitive to the component of the moment along $\boldsymbol{b}$ ) and $\Psi=-180^{\circ}$ (lower panel, sensitive to the component along $\boldsymbol{a}$ ). The intensity is plotted on a $\log$ scale.

coupling between bilayers, is uniquely consistent with our data.

To understand the temperature evolution of the magnetic structure, we developed a model in which the cycloid is decomposed into two spin-density wave components $\pi / 2$ out of phase, that in the commensurate limits are equivalent to the $\mathrm{AFM}_{\mathrm{b}}$ and $\mathrm{AFM}_{\mathrm{a}}$ structures. The only free parameters are the amplitudes of these components, $M_{b}$ and $M_{a}$, which describe the elongation of the envelope of the cycloid along the $\boldsymbol{b}$ and $\boldsymbol{a}$ axes, respectively. We fit the azimuthal dependences using MAGNETIX [solid lines in Fig. 3(b)] and found that this simple model provides a remarkably accurate description of the data at all temperatures. The fits can be intuitively understood by neglecting the small $h$ component of the wave vector, which simplifies the dependence to $\propto\left(M_{b} \sin \Psi\right)^{2}+\left(M_{a} \cos \Psi\right)^{2}$. It can then be seen that the peak-to-peak amplitude of the oscillations, $M_{b}^{2}-M_{a}^{2}$, is directly related to the eccentricity of the cycloid (after normalizing out the effect of the increase in (a)

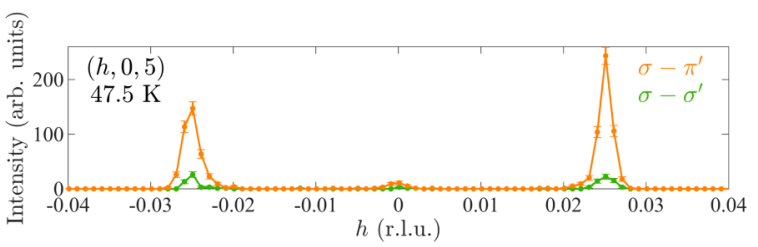

(b)

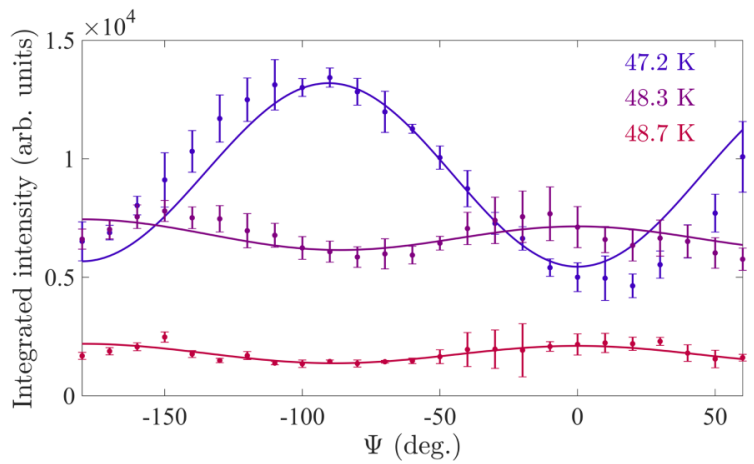

(c)

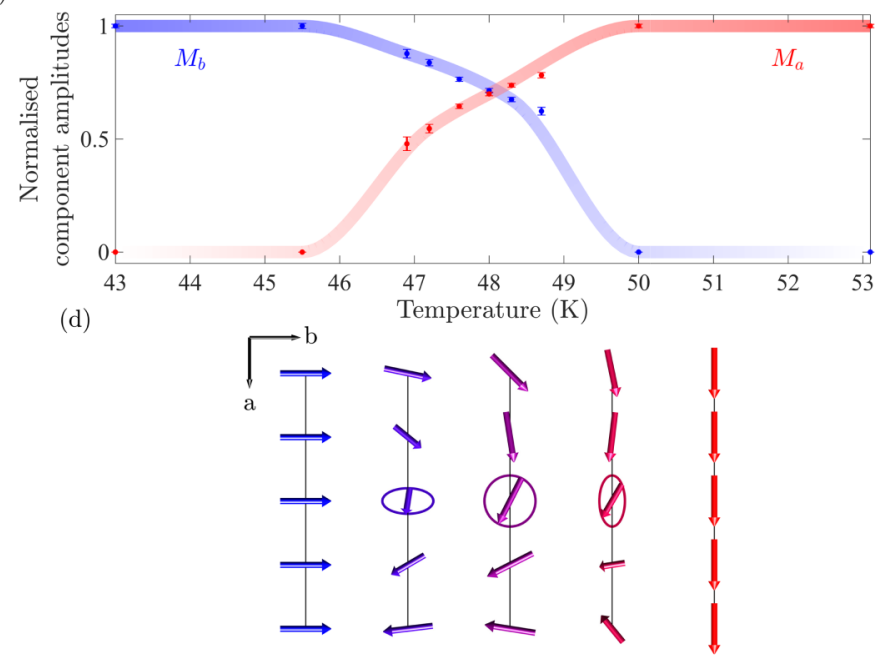

FIG. 3. Evolving cycloidal structure from resonant x-ray scattering. (a) $h$ scans through $(0,0,5)$ in both polarization channels, showing that the incommensurate satellites are magnetic. (b) Azimuthal dependences of the $(-\delta, 0,5)$ satellite at select temperatures. The solid lines are fits to the cycloidal model described in the text. (c) Amplitudes of the cycloid components extracted from fits like those in (b), normalized by $\left(M_{a}^{2}+M_{b}^{2}\right)^{-1 / 2}$ to remove the effect of the increasing moment size on cooling. Solid lines are guides to the eye. (d) Schematic of the evolution of the magnetic structure with temperature, with the lengths of the arrows indicating the changing of the cycloidal envelope from elongated along $\boldsymbol{b}$, to circular, to elongated along $\boldsymbol{a}$. The changing moment size and period of the cycloid are neglected for clarity. 
the overall moment size on cooling), while the phase depends on whether $M_{b}$ or $M_{a}$ is larger. The normalized fitted values of the amplitudes are shown in Fig. 3(c) [including fits to the commensurate dependences such as those in Fig. 2(c)]. In the commensurate phases only one of the components is present, as expected. In the incommensurate phase, by contrast, both amplitudes are finite and vary with temperature. This describes the magnetic structure shown schematically in Fig. 3(d), where the envelope of the cycloid transitions from elongated along $\boldsymbol{b}$, to circular, to elongated along $\boldsymbol{a}$. Our x-ray data have therefore revealed a complex and evolving cycloidal magnetic structure which mediates the SRT.

Theoretical justification of this model is provided by a symmetry analysis of terms in the free energy (full details can be found in the Supplemental Material [21]). The $\mathrm{AFM}_{\mathrm{b}}$ and $\mathrm{AFM}_{\mathrm{a}}$ phases can each be associated with a one-dimensional order parameter, $\mu$ and $\rho$. The polar structure of $\mathrm{Ca}_{3} \mathrm{Ru}_{2} \mathrm{O}_{7}$ allows the Lifshitz-type invariant $\mu(\partial \rho / \partial y)-\rho(\partial \mu / \partial y)$ in the free energy. Such invariants describe instabilities towards incommensurate modulated states [25,28,29], including the cycloid observed here. The microscopic origin of the Lifshitz invariant lies in a uniform DMI, which competes with easyaxis anisotropies to select the ground state of the system. Away from $T_{S}$, the easy-axis anisotropies dominate and preclude the formation of a modulated state, leading to the $\mathrm{AFM}_{\mathrm{b}}$ or $\mathrm{AFM}_{\mathrm{a}}$ phase. As the easy-axis gradually changes from $\boldsymbol{b}$ to $\boldsymbol{a}$ in the vicinity of the SRT, however, we expect minimal or easy-plane anisotropy, allowing the uniform DMI to stabilize the cycloidal phase.

The frustration that causes the evolution of the envelope and period of the cycloid with temperature should also make it highly sensitive to other perturbations. To demonstrate this, we performed a neutron scattering experiment on $\mathrm{Ca}_{3} \mathrm{Ru}_{2} \mathrm{O}_{7}$ with a magnetic field applied along the $\boldsymbol{b}$ axis. The resulting temperature-field phase diagram is shown in Fig. 4, revealing a large expansion of the cycloidal phase under small fields. A striking feature of the phase boundary is its similarity with that of the so-called "metamagnetic texture" reported by Sokolov et al. [16], who measured incommensurate peaks at $( \pm \Delta, 0,0)$ in fields above $2 \mathrm{~T}$ with small angle neutron scattering (SANS). The connection to the magnetic satellites that we observe can be seen by doubling our wave vector $(\delta, 0,1)$ and then projecting back into the first Brillouin zone through subtraction of the lattice vector $(0,0,2)$, giving $(2 \delta, 0,0) \approx(\Delta, 0,0)[30]$. It is then readily apparent that the peaks observed by Sokolov et al. are in fact second harmonics of our satellites, whose presence at zero field rule out the proposed metamagnetic texture. While we could not directly observe the second harmonic satellites in our experiment [31], their appearance under magnetic fields is naturally explained by a phase modulation of the cycloid stabilized by symmetry-allowed terms in the free energy (see the Supplemental Material [21]). This phase modulation corresponds to the spins bunching along the field direction in order to reduce their Zeeman energy, shown schematically by the purple arrows in Fig. 4. In this scenario, a net magnetization develops as higher harmonics are generated continuously from the zerofield cycloid, in the absence of any metamagnetic transition. Such behavior is reminiscent of the highly robust, tunable soliton lattices seen in chiral helimagnets under field [32].

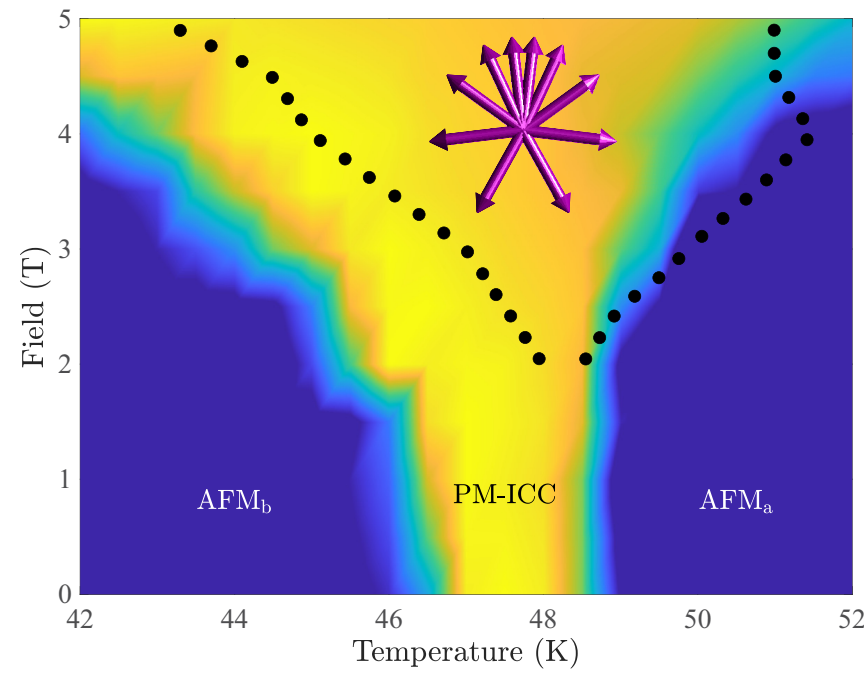

FIG. 4. Phase diagram for a magnetic field along the $\boldsymbol{b}$ axis from neutron scattering. The color scale is the integrated intensity of the $(\delta, 0,1)$ peak, with the $\mathrm{AFM}_{\mathrm{b}}, \mathrm{AFM}_{\mathrm{a}}$, and phase-modulated incommensurate cycloid (PM-ICC) phases marked. The black dotted line encloses the region over which peaks at $(\Delta, 0,0) \approx(2 \delta, 0,0)$ were seen in a previous SANS measurement [16]. The purple arrows are a cartoon of the spin distribution in the $\boldsymbol{a}-\boldsymbol{b}$ plane in the PM-ICC phase, depicted with a circular envelope for clarity.

Within this framework, we can also explain previous observations of incommensurate structures in doped $\mathrm{Ca}_{3} \mathrm{Ru}_{2} \mathrm{O}_{7}$ [17-19]. Here, a reduced anisotropy from the introduction of magnetic dopants should allow an easier turning of the moments away from their easy-axis by the DMI, stabilizing cycloidal structures with shorter repeat distances and over larger temperature ranges.

Our analysis therefore unifies previously disparate magnetic behaviors of $\mathrm{Ca}_{3} \mathrm{Ru}_{2} \mathrm{O}_{7}$, attributing them to a common origin and revealing a highly rich phase diagram. Knowledge of the mediating cycloid, and the ingredients necessary for its formation from symmetry analysis, will be vital for the development of a conclusive microscopic theory of the technologically relevant SRT. Alongside a review of bulk thermodynamic and magnetization data in the vicinity of the SRT, recent work which ascribes the reorientation to a "magnetoelectric" anisotropy [15] will need revising in light of our results. Finally, given the fragility of the cycloidal order and the strong coupling between the structural, electronic, and magnetic degrees of freedom in $\mathrm{Ca}_{3} \mathrm{Ru}_{2} \mathrm{O}_{7}$, one might imagine the possibility of using mechanical or electrical stimuli as tuning parameters, with profound applications in magnetic memory and spintronics.

We thank Frank Krüger, Andrew Green, Michal Kwasigroch, and Adam Walker for insightful discussions. We also thank Richard Thorogate for assistance with the resistivity measurement, Daniel Nye and Gavin Stenning for assistance with the powder $\mathrm{x}$-ray diffraction and Laue alignment in the Materials Characterisation Laboratory at the ISIS Neutron and Muon Source, Mike Matthews for technical support at I16, and Jacob Simms, Katherine Mordecai, Jon Bones, and David Keymer for technical support at WISH. C.D.D. was supported 
by the Engineering and Physical Sciences Research Council (EPSRC) Centre for Doctoral Training in the Advanced Characterisation of Materials under Grant No. EP/L015277/1. R.D.J. acknowledges support from a Royal Society University Research Fellowship. Work at UCL was supported by the EPSRC under Grants No. EP/N027671/1, EP/N034694/1 and No. EP/P013449/1. Experiments at the ISIS Neutron and Muon Source were supported by a beamtime allocation from the Science and Technology Facilities Council under Proposal No. RB1920210. We acknowledge the Diamond Light Source for time on beamline I16 under Proposal No. MM23580.
[1] N. A. Hill, J. Phys. Chem. B 104, 6694 (2000).

[2] N. A. Spaldin and M. Fiebig, Science 309, 391 (2005).

[3] S.-W. Cheong and M. Mostovoy, Nat. Mater. 6, 13 (2007).

[4] T. Kimura, T. Goto, H. Shintani, K. Ishizaka, T. Arima, and Y. Tokura, Nature (London) 426, 55 (2003).

[5] Y. Shi, Y. Guo, X. Wang, A. J. Princep, D. Khalyavin, P. Manuel, Y. Michiue, A. Sato, K. Tsuda, S. Yu, M. Arai, Y. Shirako, M. Akaogi, N. Wang, K. Yamaura, and A. T. Boothroyd, Nat. Mater. 12, 1024 (2013).

[6] T. H. Kim, D. Puggioni, Y. Yuan, L. Xie, H. Zhou, N. Campbell, P. J. Ryan, Y. Choi, J.-W. Kim, J. R. Patzner, S. Ryu, J. P. Podkaminer, J. Irwin, Y. Ma, C. J. Fennie, M. S. Rzchowski, X. Q. Pan, V. Gopalan, J. M. Rondinelli, and C. B. Eom, Nature (London) 533, 68 (2016).

[7] G. Cao, S. McCall, J. E. Crow, and R. P. Guertin, Phys. Rev. Lett. 78, 1751 (1997).

[8] Y. Yoshida, S.-I. Ikeda, H. Matsuhata, N. Shirakawa, C. H. Lee, and S. Katano, Phys. Rev. B 72, 054412 (2005).

[9] D. Puggioni, M. Horio, J. Chang, and J. M. Rondinelli, Phys. Rev. Research 2, 023141 (2020).

[10] S. Lei, M. Gu, D. Puggioni, G. Stone, J. Peng, J. Ge, Y. Wang, B. Wang, Y. Yuan, K. Wang, Z. Mao, J. M. Rondinelli, and V. Gopalan, Nano Lett. 18, 3088 (2018).

[11] B. Bohnenbuck, I. Zegkinoglou, J. Strempfer, C. SchüßlerLangeheine, C. S. Nelson, P. Leininger, H.-H. Wu, E. Schierle, J. C. Lang, G. Srajer, S. I. Ikeda, Y. Yoshida, K. Iwata, S. Katano, N. Kikugawa, and B. Keimer, Phys. Rev. B 77, 224412 (2008).

[12] W. Bao, Z. Q. Mao, Z. Qu, and J. W. Lynn, Phys. Rev. Lett. 100, 247203 (2008).

[13] F. Baumberger, N. J. C. Ingle, N. Kikugawa, M. A. Hossain, W. Meevasana, R. S. Perry, K. M. Shen, D. H. Lu, A. Damascelli, A. Rost, A. P. Mackenzie, Z. Hussain, and Z.-X. Shen, Phys. Rev. Lett. 96, 107601 (2006).

[14] M. Horio, Q. Wang, V. Granata, K. P. Kramer, Y. Sassa, S. Jöhr, D. Sutter, A. Bold, L. Das, Y. Xu, R. Frison, R. Fittipaldi, T. K. Kim, C. Cacho, J. E. Rault, P. Le Fèvre, F. Bertran, N. C. Plumb, M. Shi, A. Vecchione et al., arXiv:1911.12163.

[15] I. Marković, M. D. Watson, O. J. Clark, F. Mazzola, E. Abarca Morales, C. A. Hooley, H. Rosner, C. M. Polley, T. Balasubramanian, S. Mukherjee, N. Kikugawa, D. A. Sokolov, A. P. Mackenzie, and P. D. C. King, Proc. Natl. Acad. Sci. USA 117, 15524 (2020).

[16] D. A. Sokolov, N. Kikugawa, T. Helm, H. Borrmann, U. Burkhardt, R. Cubitt, J. S. White, E. Ressouche, M. Bleuel, K. Kummer, A. P. Mackenzie, and U. K. Rößler, Nat. Phys. 15, 671 (2019).

[17] X. Ke, J. Peng, W. Tian, T. Hong, M. Zhu, and Z. Q. Mao, Phys. Rev. B 89, 220407(R) (2014).

[18] M. Zhu, J. Peng, W. Tian, T. Hong, Z. Q. Mao, and X. Ke, Phys. Rev. B 95, 144426 (2017).
[19] S. Lei, S. Chikara, D. Puggioni, J. Peng, M. Zhu, M. Gu, W. Zhao, Y. Wang, Y. Yuan, H. Akamatsu, M. H. W. Chan, X. Ke, Z. Mao, J. M. Rondinelli, M. Jaime, J. Singleton, F. Weickert, V. S. Zapf, and V. Gopalan, Phys. Rev. B 99, 224411 (2019).

[20] L. C. Chapon, P. Manuel, P. G. Radaelli, C. Benson, L. Perrott, S. Ansell, N. J. Rhodes, D. Raspino, D. Duxbury, E. Spill, and J. Norris, Neutron News 22, 22 (2011).

[21] See Supplemental Material at http://link.aps.org/supplemental/ 10.1103/PhysRevB.102.180410 for further experimental details, a symmetry analysis of terms in the free energy which stabilize the cycloidal order and its harmonics under magnetic fields, and an analytical model of the phase-modulated cycloid, which includes Refs. [33-35].

[22] J. M. Tranquada, B. J. Sternlieb, J. D. Axe, Y. Nakamura, and S. Uchida, Nature (London) 375, 561 (1995).

[23] C. Lester, S. Ramos, R. S. Perry, T. P. Croft, R. I. Bewley, T. Guidi, P. Manuel, D. D. Khalyavin, E. Forgan, and S. M. Hayden, Nat. Mater. 14, 373 (2015).

[24] Y. Ishikawa, K. Tajima, D. Bloch, and M. Roth, Solid State Commun. 19, 525 (1976).

[25] I. Sosnowska and A. K. Zvezdin, J. Magn. Magn. Mater. 140144, 167 (1995).

[26] J. P. Hill and D. F. Mcmorrow, Acta Crystallogr., Sect. A 52, 236 (1996).

[27] L. Chapon, MagnetiX, https://forge.epn-campus.eu/projects/ magnetix.

[28] Y. A. Izyumov and V. Syromyatnikov, Phase Transitions and Crystal Symmetry (Springer, Dordrecht, 1990).

[29] A. M. Kadomtseva, A. K. Zvezdin, Y. F. Popov, A. P. Pyatakov, and G. P. Vorob'ev, J. Exp. Theor. Phys. Lett. 79, 571 (2004).

[30] The wave vectors of the satellite peaks are field and temperature dependent, but comparing the value from our data at $2 \mathrm{~T}$ and $47 \mathrm{~K}, \delta \approx 0.023$, with the SANS data at at the same field and temperature, $\Delta \approx 0.045$, we can see the correspondence $2 \delta \approx$ $\Delta$.

[31] Observation of the second harmonics of the satellite peaks in our neutron experiment was precluded by the low flux of longwavelength neutrons at such high $d$ spacing.

[32] Y. Togawa, T. Koyama, K. Takayanagi, S. Mori, Y. Kousaka, J. Akimitsu, S. Nishihara, K. Inoue, A. S. Ovchinnikov, and J. Kishine, Phys. Rev. Lett. 108, 107202 (2012).

[33] N. Dobelin and R. Kleeberg, J. Appl. Crystallogr. 48, 1573 (2015).

[34] O. Arnold, J. Bilheux, J. Borreguero, A. Buts, S. Campbell, L. Chapon, M. Doucet, N. Draper, R. Ferraz Leal, M. Gigg, V. Lynch, A. Markvardsen, D. Mikkelson, R. Mikkelson, R. Miller, K. Palmen, P. Parker, G. Passos, T. Perring, P. Peterson et al., Nucl. Instrum. Methods Phys. Res., Sect. A 764, 156 (2014).

[35] D. G. Porter, Py16, https://doi.org/10.5281/zenodo.3859719. 